\title{
ON SOME INVARIANTS OF MAPPINGS
}

\author{
KIYOSHI AOKI
}

(Received May 15, 1953)

1. Introduction. In the study of homotopy groups of sphere there are very few methods for determining whether a mapping of one sphere on another is essential or not. One such method is furnished by Brouwer degree of a mapping of $S^{r}$ on itself. Another is furnished by the Hopf invariant of a mapping of $S^{2 r-1}$ on $S^{\prime}[3,4]$. These methods are used only for mappings of $S^{n}$ on $S^{r}$ with $n=r$ or $n=2 r-1$. Freudenthal's results [2] are applied for the case $r<n<2 r-1$ but almost nothing are known about $\pi_{n}\left(S^{r}\right)$ for $n>2 r-1$. L. Pontrjagin [6] succeeded in the enumeration of the homotopy classes of maps of a 3-complex $K^{3}$ on $S^{2}$ and obtained the result that Hopf's invariant determines completely homotopic classes of the maps of $S^{3}$ on $S^{2}$; Whitney [8] reformulated another Hopf's theorem [5] and introduced two deformation theorems. In this paper we shall at first attempt to generalize both Hopf's invariant and Brouwer degree. Such quantity will be used for mappings of $S^{n}$ on $S^{r}$ with $n=(k+1) r-k(k=0,1,2,3, \ldots)$. Secondly for this quantity we shall attempt to generalize Pontrjagin's and some other theorems.

2. Hopf [3] studied many interesting properties of maps of $S^{3}$ on $S^{2}$ and he stated [4] the generalization of these results for the maps of $S^{2 r-1}$ on $S^{r}$ but he omitted these proofs.

We now consider maps $f$ of $S^{m}$ on $S^{n}(m>n)$ and denote by $T^{n}$ any $n$ dimensional simplex on $S^{m}$, by $\tau^{n}$ a fined $n$-dimensional simplex on $S^{n}$. Let $\xi$ be an interior point of $\tau^{n}$. When $\xi$ has only one interior point $x$ in $T^{n}$ as inverse image of $f$, we denote $\varphi_{T}^{n}(\xi)= \pm x$. The signs of $x$ will be + or according to whether $T^{n}$ is mapped on $\tau^{n}$ positively or negatively. If $\xi$ does not have inverse image of $f$ in $T^{n}$, we define $\varphi_{T^{n}}(\xi)=0$. We consider any integral complex $C^{n}=\Sigma a_{i} T_{i}^{n}$ and define by $\varphi_{C^{n}}(\xi)=\Sigma a_{i} \varphi_{T_{i}^{n}}^{n}(\xi)$ the inverse image for $C^{n}$. This integral complex is clearly 0-dimensional. From the definition the following relations are introduced:

$$
\begin{gathered}
\mathscr{P}_{1}^{n}+C_{2}^{n}(\xi)=\varphi_{C_{1}^{n}}(\xi)+\boldsymbol{\varphi}_{C_{2}^{n}}(\xi) \\
\boldsymbol{\varphi}_{-C^{n}}(\xi)=-\boldsymbol{\varphi}_{C^{n}}(\xi) \\
\boldsymbol{\varphi}_{0}(\xi)=0
\end{gathered}
$$

Secondly we consider any $r$-dimensional simplex $(m \geqq r \geqq n+1) T^{r}$ and denote by $\varphi_{T^{r}}(\xi)$ the intersection of the inverse image of $\xi$ and $T^{r}$. From the definition the following relations are introduced:

$$
\begin{aligned}
& \varphi_{C_{1}^{r}+C_{2}^{r}}(\xi)=\phi_{C_{1}^{r}}(\xi)+\boldsymbol{\phi}_{c_{2}^{r}}(\xi) \\
& \varphi_{-C^{r}}(\xi)=-\boldsymbol{\varphi}_{C^{r}}(\xi)
\end{aligned}
$$




$$
\begin{aligned}
& \boldsymbol{\varphi}_{0}(\xi)=0 \\
& \dot{\varphi}_{c^{r}}(\xi)=\varphi_{\dot{c}} r(\xi)
\end{aligned}
$$

From the property $\dot{S}^{m}=0$ and $\left(3^{\prime}\right),\left(4^{\prime}\right)$, we know $\dot{\varphi}_{S^{m}}(\xi)=0$. On the other hand $S^{m}$ is a manifold and $\varphi_{S^{m}}(\xi)$ consists of finite numbers of closed manifolds $M_{1}^{m-n}, M_{2}^{m-n}, \ldots M_{k}^{-n m}$ which are disjoint each other. If we apply the Freudenthal's Lemma [2] for our mappings, we may assume $k=1$ without any loss of generality. We consider $r+1$ points $\xi_{0}, \xi_{1}, \ldots \xi_{r}$ on $S^{n}$ and denote by $M_{i}^{m-n}$ the inverse image of $\xi_{i}$. Let $K^{n}, \stackrel{*}{\kappa^{n}}$ be simplicial subdivision of $S^{m} S^{n}$ respectively. We introduce the standard map which has at first been introduced by Whitney and which the author has defined for more general case[1]. The following Lemma is the immediate result from the definition. Its proof is similar to the one of the preceding paper [1].

LEMMA 2.1 Let $f$ be a map of $S^{m}$ into $S^{n}$, then there exists a standard map which is homotopic to $f$.

As $M_{i}^{m-n} \sim 0$ in $S^{m}$, there exists a complex $K_{i}^{m-n+1}$ bounded by $M_{i}^{m-n}$. We shall take useful one as $K_{i}^{m-n+1}$ and following Lemma is used in studing the special one of $K^{i m-n+1}$.

LEMMA 2.2. Let $Z^{p}, Z^{q}$ be any manifold of Euclidean space $R^{m}$ which are fremed each other. The regular connected complexes which are bounded by $Z^{p}$, $Z^{q}$ respectively can be deforwed so as to hold at most $p+q-m+2$ dimensionalsimplexes in common.

Proof. We can assume $p>q$ without any loss of generality. Let $K^{p+1}$, $K^{q+1}$ be regularly connected complexes bounded by $Z^{p}, Z^{q}$ respectively and holding in common $(q+1)$-dimensional simplex $\left(a_{0}, a_{1}, \ldots a_{q+1}\right)$. If $q+1 \leqq$ $\boldsymbol{p}+q-\boldsymbol{m}+2$, then this Lemma is evident. Thus we assume $q+1>p+q$ $-\boldsymbol{m}+2$ and consider $(q+2)-\operatorname{simplex}\left(a_{0}, a_{1}, \ldots, a_{q+9}\right)$ and its interior point b. We replace the simplex $\left(a_{0}, a_{1}, \ldots, a_{q+1}\right)$ of $K^{q+1}$ by

$\left[\left(b a_{1} \ldots a_{q+1}-\left(b a_{0} a_{2} \ldots a_{q+1}\right)+\ldots .+(-1)^{n+1}\left(b a_{0} a_{1} \ldots a_{q}\right)\right]\right.$.

If this process is done for every common $(q+1)-$ simplex of $K^{p+1}$ and $K^{4+1}$, then they have common simplexes which are at most $q$-dimensional. If $q=p+q-m+2$, this Lemma was completely proved. Thus we assume $q>\boldsymbol{p}+\boldsymbol{q}-m-2$. Let $\left(a_{0} a_{1} \ldots a_{q}\right)$ be any common simplex of $K^{p+1}$ and $\left(a_{0} a_{1}\right.$ $\left.\ldots a_{q} a_{q+1}\right),\left(a_{0} a_{1} \ldots a_{q} a_{q+1}^{\prime}\right)$ be a pair of $(q+1)-$ simplexes of $K^{q+1}$ which have the common $q$-simplex $\left(a_{0} a_{1} \ldots a_{q}\right)$. We consider their interior points $b, b^{\prime}$ respectively. At first we replace $\left(a_{0} a_{1} \ldots a_{q} a_{q+1}\right),\left(a_{0} a_{1} \ldots a_{q} a_{q+1}^{\prime}\right)$ by

$$
\begin{aligned}
& C_{1}^{q+1}=\left[\left(b a_{1} \ldots a_{q} a_{q+1}\right)-\left(b a_{0} a_{2} \ldots a_{q} a_{q+1}\right)+\ldots+(-1)^{q}\left(b a_{0} a_{1} \ldots\right.\right. \\
& C_{2}^{q+1}=\left[\left(b^{\prime} a_{1} \ldots a_{q} a_{q+1}^{\prime}\right)-\left(b^{\prime} a_{0} a_{2} \ldots a_{q} a_{q+1}^{\prime}\right)+\ldots+(-1)^{q}\left(b a_{0} a_{1} \ldots \ldots .\right.\right.
\end{aligned}
$$

respectively. As $q>p+q+2-m$, we can construct regularly connected complex $C^{q+1}$ bounded by

$$
\left[\left(b a_{1} \ldots a_{q}\right)-\left(b a_{0} a_{2} \ldots a_{q}\right)+\ldots+(-1)^{q}\left(b a_{\theta} a_{1} \ldots a_{q-1}\right)\right]
$$


and $\left[\left(b^{\prime} a_{1} \ldots a^{\imath}\right)-\left(b^{\prime} a_{0} a_{2} \ldots a_{q}\right)+\ldots+(-1)^{\urcorner}\left(b^{\prime} a_{0} a_{1} \ldots a_{q-1}\right)\right]$

which have common simplexes at most of $(q-1)$-dimensions with $K^{p+1}$. We replace $\left(a_{0} a_{1} \ldots a_{q} a_{q+1}\right)+\left(a_{0} a_{1} \ldots a_{1} a_{1+1}^{\prime}\right)$ by $C_{1}^{q+1}+C^{q+1}+C_{2}^{\prime+1}$. If such a process is done for every common $q$-simplexes of $K^{p+1}$ and $K^{q+1}$, then they have common simplexes at most of $(q-1)$-dimensions. If we take care of only fact that every simplex at most of $(q-1)$-dimensions is a common one face of some $(q+1)$-simplexes (its number need not to be two for the common $(q-1)$-simplex)), we can perform similar process. By a repetition of similar processes, we can lower the dimension of common simplexes of $K^{p+1}$ and $K^{1+1}$ untill at most $p+q+2-m$, where the dimension of the last common simplex is calculated from the dimensions of $S^{m}, K^{p+1}$ and $K^{q+1}$.

LEMMA 2.3. Let $Z^{p}$ be any manifold of Euclidean space $R^{m}$. If we construct a complex projecting $Z^{p}$ from a fix point, the resulted $(p+1)$-dimensional conplex may be deformed so as to have singular simplexes [1] at most of (2p $+2-m$ )-dimension.

Proof. By the similar deformations of Lemma 2.2, we can prove immediately.

THEOREM 2.1. $K_{i}^{m-n+1}$ may be chosen as a manifold which has some singular simplexes at most of $(m-2 n+2)$-dimension.

Proof. Let us consider that a fixed point of $S^{m}$ is a point at infinity, then $S^{m}$ may be regarded as the sum of the point at infinity and a $m$-dimensional Euclidean space $R^{m}$. Of course, we don't take the point at infinity on $M_{i}^{m-n}$. We project $M_{i}^{m-n}$ from a suitable point $O$ and denote by $\left[O, M_{i}^{m-n}\right]$ the resulted sets. $\left[O, M_{i}^{m-n}\right]$ are special complexes bounded by $M_{i}^{m-n}$ and are one of $K_{l}^{m-n+1}$. By Lemma 2.3, the dimension of singular simplex of [O , $\left.\boldsymbol{M}_{i}^{n-n}\right]$ is at most

$$
2(m-n+1)-m=m-2 n+2 .
$$

LEMMA 2.4. $\bigcap_{i=1}^{r}\left[O, M_{i}^{m-n}\right]$ is a sum of finite manifolds which are at most of $(m-r n+r)$-dimension having some singular simplexes at most of $[m-$ $(r+1) n+r+1]$-dimension.

Proof. By $\xi_{i} \neq \xi_{j}(i, j=0,1,2, \ldots, r, i \neq j)$, we know that

$$
M_{i}^{m-n} \bigcap M_{j}^{n-n}=0(i, j=0,1,2, \ldots r, i \neq j)
$$

If we consider $\left[O, M_{2}^{m-n}\right]$ and $\left[O, M_{2}^{m-n}\right]$ except for singular simplexes, $[O$, $\left.\boldsymbol{M}_{1}^{m-n}\right] \bigcap\left[O, \boldsymbol{M}_{2}^{m-n}\right]$ are of at most of dimensions

$$
2(m-n+1)-m=m-2 n+2 \text {. }
$$

By Theorem 2.1, the singular simplexes of $\left[O, M_{i}^{n-n}\right]$ are at most of $(m-$ $2 n+2$ )-dimensions. Therefore the dimensions of singular simplexes of $[O$, $\left.M_{1}^{m-n}\right] \cap\left[O, M_{2}^{m-n}\right]$ are at most of dimensions 


$$
(m-2 n+2)+(m-n+1)-m=m-3 n+3 .
$$

On account of singularity of $\left[O, M_{i}^{m-n}\right],\left[O, M_{1}^{m-n}\right] \cap\left[O, M_{2}^{m-n}\right]$ are a sum of some manifolds having singular simplexes.

By Lemma 2.2 and Theorem 2.1, the intersection of $\left[O, M_{1}^{m-n}\right] \cap[O$, $\left.\boldsymbol{M}_{2}^{m-n}\right]$ and $\left[O, \boldsymbol{M}_{3}^{m-n}\right]$ is studied. As the general case $\bigcap_{i=1}^{r}\left[O, \boldsymbol{M}_{i}^{m-n}\right]$ are a sum of some manifolds whose dimension are at most

$$
r(m-n+1)-(r-1) m=m-r n+r,
$$

where the dimensions of those singular simplexes are at most

$(m-2 n+2)+(r-1)(m-n+1)-(r-1) m=m-(r+1) n+r+1$.

Lemma $2.5 M_{k}^{m-n} \cap\left\{\bigcap_{i=1}^{k-1}\left[O, M_{i}^{m-n}\right]\right\} \cap\left\{\bigcap_{j=k+1}^{r}\left[O, M_{j}^{m-n}\right]\right\}$ are a sum of finite manifolds which are at most of $(m-r n+r-1)$-dimensions having some singular simplexes at most of $(m-(r+1) n+r)$-dimension.

ProOF. If we replace $r$ by $r-1$ in Lemma 2.4, $\left\{\bigcap_{i=1}^{l i-1}\left[O, M_{i}^{n_{2-n}}\right]\right\} \cap$ $\left\{\bigcap_{j=k+1}^{r}\left[O, M_{j}^{n-n}\right]\right\}$ are at most of $[m-(r-1) n+(r-1)]$-dimensions having some singular simplexes at most of $[m-r n+r]$-dimensions. Therefore the dimension of $M_{k}^{m-n} \cap\left\{\bigcap_{i=1}^{k-1}\left[O, M_{i}^{m-n}\right]\right\} \cap\left\{\bigcap_{j=k+1}\left[O, M_{j}^{n-n}\right]\right\}$ is at most $[m-$ $(r-1) n+(r-1)]+(m-n)-m=m-r n+r-1$.

The dimensions of the singular simplexes of those complexes are at most

$$
[m-r n+r]+(m-n)-m=n-(r+1) n+r .
$$

LEMMA 2.6. If $m=(r+1) n-r$, the intersection number $\phi\left(M_{0}^{m-n}, \bigcap_{i=1}^{r}[O\right.$, $\left.\left.M_{i}^{m-n}\right]\right)$ can be defined uniquely.

Proof. For $\bigcap_{i=1}^{r}\left[O, M_{i}^{m-n}\right]$ of Lemma 2. 4, we give an orientation as follows : We know an orientation for the faces of simplexes of $\left[O, M_{1}^{m-n}\right]$ and by this orientation we introduce an orientation on $\bigcap_{i=1}^{n}\left[O, M_{i}^{m-n}\right]$. This orientation does not depend upon the singular simplexes of $\bigcap_{i=1}^{r}\left[O, M_{i}^{m-n}\right]$. Similarly we introduce an orientation for $M_{k}^{m-n} \cap\left\{\bigcap_{i=0,1,2, \ldots, \hat{k}, . . r}\left[O, M_{i}^{m-n}\right]\right\}$ by the faces of simplexes of $M_{k}^{m-n}$. This orientation does not depend upon the singular 
simplexes of $M_{k}^{m-n} \cap\left\{\bigcap_{i=0,1,2, \ldots, \hat{k}, \ldots r}\left[O, M_{1}^{m-n}\right]\right.$. Then, by lemma 2.4,

$$
\operatorname{dim}\left\{\bigcap_{i=1}^{r}\left[O, M_{i}^{n-n}\right]\right\}=m-r n+r=n .
$$

The intersection of $M_{0}^{m-n}$ and $\bigcap_{i=1}^{r}\left[O, M_{0}^{m-n}\right]$ are at most of dimensions $n+$ $(m-n)-m=0$.

From the orientations of $R^{m}, M_{0}^{m-n}$ and $\bigcap_{i=1}^{r}\left[O, M_{i}^{m-n}\right]$ we know the intersection number $\phi\left(M_{0}^{m-n}, \bigcap_{i=1}^{r}\left[O, M_{i}^{m-n}\right]\right)$.

Lemma 2. 7. $\phi\left(M_{0}^{n-n}, \bigcap_{i=1}^{r}\left[O, M_{i}^{m-n}\right]\right)$ does not depend on a choice of $a$ fixed point $O$.

PROoF. We consider $m$-dimensional Euclidean space $R^{m}$ as in the proof of Theorem 2.1 and fixed point $O^{\prime}$ which is in $R^{m}-\bigcup_{i} M_{i}^{m-n}-O$.

By the projection from $O^{\prime}$, we get a similar complex $\left[O^{\prime}, M_{i}^{m-n}\right]$. If the bounded set containing $O$ and $O^{\prime}$ are covered by sufficiently fine open set, we can choose a finite covering, which refine the given covering. If we consider point-pairs in the same element of covering at first and remove from one element to the adjacent secondly, we can remove from $O$ to $O^{\prime}$ by finite processes. Therefore we can assume without any loss of generality that $O^{\prime}$ exist in sufficiently small neighborhood of $O$. We shall prove

$$
\phi\left(M^{m-n}, \bigcap_{i=1}^{r}\left[O, M_{i}^{m-n}\right]\right)=\phi\left(M_{0}^{m-n}, \bigcap_{i=1}^{r}\left[O^{\prime}, M_{i}^{m-n}\right]\right) .
$$

As $O$ and $O^{\prime}$ lie in sufficiently near, for $k=0,1, M_{i}^{m-n} \cap\left(\bigcap_{i=2}^{r}\left[O, M_{i}^{m-n}\right]\right)$ and $M_{i}^{m-n} \cap\left(\bigcap_{i=2}^{r}\left[O^{\prime}, M_{i}^{m-n}\right]\right)$ are situated sufficiently near in $R^{m}$ and are fremed each other. We consider a complex $X$ which is bounded by $\boldsymbol{M}_{0}^{m-i}$ $\cap\left(\bigcap_{i=2}^{r}\left[O, M_{i}^{m-n}\right]\right)-M_{j}^{m-n} \cap\left(\bigcap_{i=2}^{r}\left[O, M_{i}^{m-n}\right]\right)$ and is fremed from $M_{1}^{m-n} \cap$ $\left(\bigcap_{i=2}^{r}\left[O, M_{i}^{m-n}\right]\right)$ and $M_{1}^{m-n} \cap\left(\bigcap_{i=2}^{r}\left[O^{\prime}, M_{i}^{m-n}\right]\right)$. Similarly we consider a complex $Y$ which is bounded by $M_{1}^{m-n} \cap\left(\bigcap_{i=2}^{r}\left[O, M_{i}^{m-n}\right]\right)-M_{1}^{m-n} \cap\left(\bigcap_{i=2}^{r}\right.$ 
$\left.\left[O^{\prime}, M_{i}^{m-n}\right]\right)$ and is fremed from $M_{0}^{m-n} \cap\left(\bigcap_{i=2}^{r}\left[O, M_{i}^{m-n}\right]\right)$ and $M_{0}^{m-n} \cap\left(\bigcap_{i=2}^{r}\right.$ $\left.\left[O, M_{i}^{m-n}\right]\right)$.

$$
\begin{aligned}
& R \partial\left\{\left[O, M_{1}^{m-n}\right] \cap\left(\bigcap_{i=2}^{r}\left[O, M_{i}^{m-n}\right]\right)\right\}=M_{1}^{m-n} \cap\left(\bigcap_{i=2}^{r}\left[O, M_{i}^{m-n}\right]\right), \\
& R \partial\left\{\left[O^{\prime}: M_{1}^{m-n}\right] \cap\left(\bigcap_{i=2}^{r}\left[O^{\prime}, M_{i}^{m-n}\right]\right)\right\}=M_{1}^{m-n} \cap\left(\bigcap_{i=2}^{r}\left[O^{\prime}, M_{i}^{m-n}\right]\right) .
\end{aligned}
$$

Then

- $R \partial\left\{\left[O, M_{1}^{m-n}\right] \cap\left(\bigcap_{i=2}^{r}\left[O, M_{i}^{m-n}\right]\right)-Y-\left[O^{\prime}, M_{1}^{m-n}\right] \cap\left(\bigcap_{i=2}^{r}\left[O^{\prime}, M_{i}^{m-n}\right]\right)=0\right.$,

$$
\begin{gathered}
\phi\left\{M_{0}^{m-n} \cap\left(\bigcap_{i=2}^{r}\left[O, M_{i}^{m-n}\right]\right),\left[O, M_{1}^{m-n}\right] \cap\left(\bigcap_{i=2}^{r}\left[O, M_{i}^{m-n}\right]\right)-Y-\right. \\
\left.\left[O^{\prime}, M_{1}^{m-n}\right] \cap\left(\bigcap_{i=2}^{r}\left[O^{\prime}, M_{i}^{m-n}\right]\right)\right\}=0 .
\end{gathered}
$$

On the other hand,

$$
\begin{aligned}
& \phi\left(M_{0}^{m-n}, \bigcap_{i=1}^{r}\left[O, M_{i}^{m-n}\right]\right) \\
= & \varepsilon \phi\left(M_{0}^{m-n} \cap\left(\bigcap_{i=2}^{r}\left[O, M_{i}^{m-n}\right]\right),\left[O, M_{1}^{m-n}\right] \cap\left(\bigcap_{i=2}^{r}\left[O, M_{i}^{m-n}\right]\right)\right. \\
\phi & \left\{M_{0}^{m-n} \cap\left(\bigcap_{i=2}^{r}\left[O, M_{i}^{m-n}\right], Y\right\}=0 . \quad(\varepsilon= \pm 1)\right.
\end{aligned}
$$

Therefore,

$$
\begin{aligned}
& \phi\left\{M_{0}^{m-n} \cap\left(\bigcap_{i=2}^{r}\left[O, M_{i}^{m-n}\right]\right),\left[O, M_{1}^{m-n}\right] \cap\left(\bigcap_{i=2}^{r}\left[O, M_{i}^{m-n}\right]\right)\right\} \\
= & \phi\left\{M_{0}^{m-n} \cap\left(\bigcap_{i=2}^{r}\left[O, M_{i}^{m-n}\right],,\left[O^{\prime}, M_{1}^{m-n}\right] \cap\left(\bigcap_{i=2}^{r}\left[O^{\prime}, M_{i}^{m-n}\right]\right)\right\} .\right.
\end{aligned}
$$

Similarly,

$$
\begin{aligned}
& \phi\left\{\left[O, M_{0}^{m-n}\right] \cap\left(\bigcap_{i=2}^{r}\left[O, M_{i}^{m-n}\right]\right), M_{1} \cap\left(\bigcap_{i=2}^{r}\left[O^{\prime}, M_{i}^{m-n}\right]\right)\right\} \\
= & \phi\left\{\left[O^{\prime}, M_{0}^{m-n}\right] \cap\left(\bigcap_{i=2}^{r}\left[O^{\prime}, M_{i}^{m-n}\right]\right), M_{1} \cap\left(\bigcap_{i=2}^{r}\left[O^{\prime}, M_{i}^{m-n}\right]\right)\right\} .
\end{aligned}
$$

Then

$$
\begin{aligned}
& \phi\left\{M_{0}^{m-n} \cap\left(\bigcap_{i=2}^{r}\left[O, M_{i}^{m-n}\right]\right),\left[O, M_{1}^{m-n}\right] \cap\left(\bigcap_{i=2}^{r}\left[O, M_{i}^{m-n}\right]\right)\right\} \\
& =\phi\left\{M_{0}^{m-n} \cap\left(\bigcap_{i=2}^{r}\left[O, M_{i}^{m-n}\right]\right),\left[O^{\prime}, M_{1}^{m-n}\right] \cap\left(\bigcap_{i=2}^{r}\left[O^{\prime}, M_{i}^{m-n}\right]\right)\right\}
\end{aligned}
$$




$$
\begin{aligned}
= & (-1)^{n-1} \phi\left\{\left[O, M_{0}^{m-n}\right] \cap\left(\bigcap_{i=2}^{r}\left[O, M_{i}^{m-n}\right]\right), M_{1}^{m-n} \cap\left(\bigcap_{i=2}^{r}\left[O^{\prime}, M_{i}^{m-n}\right]\right)\right\} \\
= & (-1)^{n-1} \phi\left\{\left[O^{\prime}, M_{0}^{m-n}\right] \cap\left(\bigcap_{i=2}^{r}\left[O^{\prime}, M_{i}^{m-n}\right]\right), M_{1}^{m-n} \cap\left(\bigcap_{i=2}^{r}\left[O^{\prime}, M_{i}^{m-n}\right]\right)\right\} \\
= & \phi\left\{M_{0}^{m-n} \cap\left(\bigcap_{r=2}^{i}\left[O^{\prime}, M_{i}^{m-n}\right]\right),\left[O^{\prime}, M_{1}^{m-n}\right] \cap\left(\bigcap_{i=2}^{r}\left[O^{\prime}, M_{i}^{m-n}\right]\right)\right. \\
= & \frac{1}{\varepsilon} \phi\left\{M_{0}^{m-n}, \bigcap_{i=1}^{r}\left[O^{\prime}, M_{i}^{m-n}\right]\right\}, \\
& \phi\left(M_{0}^{m-n}, \bigcap_{i=1}^{r}\left[O, M_{i}^{m-n}\right]\right)=\phi\left(M_{0}^{n-n}, \bigcap_{i=1}^{r}\left[O^{\prime}, M_{i}^{m-n}\right]\right),
\end{aligned}
$$

Definition 2.1. When $m=n$, we define $\phi\left[M_{0}^{0}, \theta\right]$ ( $\theta$ means the empty set) as follows ;

Let $t_{+}^{0}$, or $t^{0}$ - be the numbers of simplexes of $K^{n n}$ which are mapped on $\tau_{0}^{n}$ positively or negatively respectively. Then

$$
\phi\left[M_{0}^{0}, \theta\right]=t_{+}^{0}-t_{-}^{0} \text {. }
$$

By Lemma 2.7 we know that $\phi\left\{M_{0}^{m-n}, \bigcap_{i=1}^{r}\left[O, M_{i}^{m-n}\right]\right\}$ does not depend on a choice of a fixed point $O$. By these reason and definition 2.1 we define as follows:

Definition 2.2. In cases $m=(r+1) n-r$ or $m n$ :

$$
\left.W_{r}\left(f, \xi_{0}, \xi_{1}, \ldots, \xi_{r}\right)=\left[\phi\left\{M_{0}^{m-n}, \bigcap_{i=1}^{r}\left[O, M_{i}^{m-n}\right]\right\}\right]^{r}, W_{0}\left(f, \xi_{0}\right)=\phi \mid M_{0}^{0}, \theta\right] .
$$

LEMMA 2. 8. $W_{r}\left(f, \xi_{0}, \xi_{1}, \ldots, \xi_{r}\right)$ does not depend on a choice of $\xi_{0}, \xi_{1}, \ldots$, $\xi$. in the following cases:

(i) $m=n$, or

(ii) $m=(r+1) n-r$ and $n$ is even.

Proof. In case (i) $W_{r}\left(f, \xi_{0}\right)$ means the Brouwer degree, and Lemma 2.8 is well known.

We shall prove this Lemma in case (ii).

$$
\begin{aligned}
& \phi\left(M_{0}^{m-n}, \bigcap_{i=1}^{r}\left[O, M_{i}\right]\right)=\varepsilon \phi\left\{M_{0}^{m-n} \cap\left(\bigcap_{i=2}^{r}\left[O, M_{i}\right]\right),\left[O, M_{1}\right]\left(\bigcap_{i=2}^{r}\left[O, M_{i}\right]\right)\right\} \\
& =(-1)^{n} \varepsilon \phi\left\{\left[O, M_{0}\right] \cap\left(\bigcap_{i=2}^{r}\left[O, M_{i}\right]\right), M_{1} \cap\left(\bigcap_{i=2}^{r}\left[O, M_{i}\right]\right)\right\} \\
& =(-1)^{n}(-1)^{n(n-1)} \varepsilon \phi\left\{M_{1} \cap\left(\bigcap_{i=2}^{r}\left[O, M_{i}\right]\right),\left[O, M_{0}\right] \cap\left(\bigcap_{i=2}^{r}\left[O, M_{i}\right]\right)\right\} \\
& =(-1)^{n}(-1)^{n(n-1)} \phi\left\{M_{1}^{n-n},\left[O, M_{0}\right] \cap\left(\bigcap_{i=2}^{r}\left[O, M_{i}\right]\right)\right\} .
\end{aligned}
$$


As $n$ is even,

$$
\begin{gathered}
W_{r}\left(f, \xi_{0}, \xi_{1}, \ldots, \xi_{r}\right)=W_{r} \cdot\left(f, \xi_{1}, \xi_{0}, \ldots, \xi_{r}\right) \ldots \ldots \ldots \ldots \ldots \ldots \\
\phi\left(M_{0}^{m-n}, \bigcap_{i=1}^{r}\left[O, M_{i}^{m-n}\right]\right) \text { means a degree of } f \mid \bigcap_{i=1}^{r}\left[O, M_{i}^{n-n}\right], \text { then } \\
W_{r}\left(f, \xi_{0}, \ldots, \xi_{r}\right) \text { does not depend on a choice of } \xi_{0} . \text { Then } \\
W_{r}\left(f, \xi_{0}, \ldots, \xi_{1}, \xi_{r}\right)=W_{r}\left(f, \xi_{1}^{\prime}, \xi_{1}, \ldots, \xi_{r}\right) \ldots \ldots \ldots \ldots \ldots \ldots
\end{gathered}
$$

By using of (1) and (2), we can introduce the following calculations :

$$
\begin{aligned}
& W_{r}\left(f, \xi_{0}, \xi_{1}, \ldots, \xi_{i}, \ldots, \xi_{r}\right)=\varepsilon W_{r}\left(f, \xi_{0}, \xi_{i}, \xi_{2}, \ldots, \xi_{1}, \ldots, \xi_{r}\right) \\
= & \varepsilon W_{r}\left(f, \xi_{i}, \xi_{0}, \xi_{2}, \ldots, \xi_{1}, \ldots, \xi_{r}\right) \\
= & \varepsilon W_{r}\left(f, \xi_{i}^{\prime}, \xi_{0}, \xi_{:}, \ldots, \xi_{1} \ldots, \ldots, \xi_{r}\right) \quad(\varepsilon= \pm 1) \\
= & W_{r}\left(f, \xi_{1}, \xi_{0}, \xi_{2}, \ldots, \xi_{i}^{\prime}, \ldots, \xi_{r}\right) \\
= & W_{r}\left(f, \xi_{0}, \xi_{1}, \xi_{2}, \ldots, \xi_{i}^{\prime}, \ldots \xi_{r}\right),
\end{aligned}
$$

that is,

$$
W_{r}\left(f, \xi_{1}, \xi_{1}, \ldots, \xi_{i}, \ldots, \xi_{r}\right)=W_{r}\left(f, \xi_{i}, \xi_{1}, \ldots, \xi_{i}^{\prime}, \ldots, \xi_{r}\right)
$$

Furthermore,

$$
\begin{aligned}
& W_{r}\left(f, \xi_{0}, \xi_{1}, \ldots, \xi_{i}^{\prime}, \ldots, \xi_{r}\right)=W_{r}\left(f, \xi_{i}, \xi_{i}, \ldots, \xi_{i}, \ldots, \xi_{r}\right) \\
= & W_{r}\left(f, \xi_{i}, \xi_{1}, \ldots \xi_{0}, \ldots \xi_{b}\right)
\end{aligned}
$$

From this relation, we can introduce the following relation by using of (3):

$W_{r}\left(f, \xi_{0}, \xi_{1}, \ldots, \xi_{i}, \ldots, \xi_{r}\right)=W,\left(f, \xi_{i}, \xi_{1}, \ldots, \xi_{0}, \ldots, \xi_{r}\right)$.

When we use (1), (2) and (4), successively,

$$
W_{r}\left(f, \xi_{0}, \xi_{1}, \ldots, \xi_{r}\right)=W_{r}\left(f, \xi_{0}^{\prime}, \xi_{1}^{\prime}, \ldots, \xi_{r}^{\prime}\right) .
$$

By Lemma 2.8 we know that $W_{r}\left(f, \xi_{0}, \xi_{1}, \ldots, \xi_{r}\right)$ does not depend on a choise of $\xi_{1}, \xi_{1}, \ldots, \xi_{r}$. Then we define as follows.

DeFinition 2.3. $W_{r}(f)=W_{r}\left(f, \xi_{0}, \xi_{1}, \ldots, \xi_{r}\right)$.

THeOREM 2.2. Let $m=n$ or $m=(r+1) n-r$ and $n$ be even. If $f, g$ are maps of $S^{n}$ into $S^{n}$ and $f$ is homotopic to $g$, then $W_{\text {. }}(f)$ equals to $\boldsymbol{W}_{\boldsymbol{r}}(g)$.

Proof. When $K^{n}, \stackrel{*}{K}^{n}$ are simplicial subdivision of $S^{m}, S^{n}$ respectively, we may assume that $f$ and $g$ are simplicial maps without any loss of generality. As $f$ is homotopic to $g$, there exist a shar of maps $f_{r}$. $(1 \leqq r \leqq$ 2 ), of $K^{n}$ into $K^{n}$, where $f_{1}=f$ and $f_{2}=g$. Then we can consider $f_{r}$ to be a map of $S^{n} \times I$ into $S^{n}$ and use it as $F(x, r)$. We define $S^{m} \times(1)=S_{1}^{m}, S^{m} \times$ (2) $=S^{n}$.

$f_{r}(x)$ also may be assumed as simplicial map. Let $\xi_{1}$ a interior point of fixed $n$-simplex $\tau_{0}^{n}$ of $K^{n}$ and $\phi_{S^{m} \times I}\left(\xi_{1}\right), \varphi_{S_{1}^{m}}^{m}\left(\xi_{1}\right), \varphi_{S_{2}^{m}}\left(\xi_{1}\right)$ inverse images of $\xi_{1}$ for $f_{r}, f, g$ respectively.

As we have $\left(S^{m} \times \dot{\mathrm{I}}\right)=S_{2}^{m}-S_{1}^{m}$, by $(1)^{\prime},(2)^{\prime}$ in $\S 2$,

$$
\phi_{S^{m} \times \dot{\mathrm{I}}}\left(\xi_{1}\right)=\phi_{S_{2}^{m}}\left(\xi_{1}\right)-\phi_{S_{1}^{m}}\left(\xi_{1}\right)
$$

We denote $\varphi_{S_{2}^{m}}^{m}\left(\xi_{1}\right), \varphi_{S_{1}^{m}}^{m}\left(\xi_{1}\right)$ by $M_{i}, M_{1}^{\prime}$ respectively. When we fix $r$, we denote the inverse image of $\xi_{1}$ for $f_{r}$ by $\varphi_{S^{m} \times(r)}\left(\xi_{1}\right)$, then 


$$
\varphi_{S^{n} \times 1}\left(\xi_{1}\right)=\bigcup_{1 \leqq r \geqq 2} \varphi_{S^{m} \times(r)}\left(\xi_{1}\right)
$$

Let $O, O^{\prime}$ be fixed points of $S^{m} \times(1), S^{m} \times(2)$ respectively. We connect $O$ with $O^{\prime}$ by an arc in $S^{m} \times I$ which intersect with $S^{m} \times(r)$ at only one point. These intersections will be denoted by $O_{r}$.

In $S^{m} \times(r)$ we project $\phi_{S^{n} \times(r)}\left(\xi_{1}\right)$ from $O_{r}$ and denote the resulted complex by $\left[O_{r}, \varphi_{S^{n} \times(r)}\left(\xi_{1}\right)\right]$. Then,

$$
\left\{\bigcup_{1 \leqq r \leqq 2}\left[O_{r}, \varphi_{S^{m} \times(r)}\left(\xi_{1}\right)\right]\right\}=\left[O, M_{1}\right]-\left[O^{\prime}, M_{1}^{\prime}\right]-\varphi_{S^{m} \times \mathrm{i}}\left(\xi_{\mathrm{i}}\right)
$$

We denote this relation by $\dot{C}_{2}=\left[O, M_{1}\right]-\left[O^{\prime}, M_{1}^{\prime}\right]-A_{2}$ and a second interior point of $\tau_{0}^{n}$ by $\xi$, then

$$
\dot{\phi}_{c_{2}}\left(\xi_{2}\right)=\phi_{\left[O, M_{1}\right]}\left(\xi_{2:}\right)-\varphi_{\left[0^{\prime}, M_{1}^{\prime}\right]}\left(\xi_{2}\right)-\phi_{A_{2}}\left(\xi_{2}\right) \text {. }
$$

As $f_{r}$ has an uniquely determined image, $\phi_{A_{2}}\left(\xi_{2}\right)=0$ (for $\xi_{1} \neq \xi_{2}$ ).

We denote $\varphi_{\left[0, M_{1}\right.}\left(\xi_{2}\right), \varphi_{\left[0^{\prime}, M_{1}^{\prime}\right]}\left(\xi_{2}\right)$ by $M_{2}, M_{1}^{\prime}$ respectively. Then

$$
\dot{\varphi}_{c_{2}}\left(\xi_{2}\right)=M_{2}-M_{2}^{\prime}
$$

$\left[O, M_{1}\right] \cap\left[O, M_{2}\right]$ and $\left[O^{\prime}, M_{1}^{\prime}\right] \cap\left[O^{\prime}, M_{2}^{\prime}\right]$ are complexes bounded by $\boldsymbol{\varphi}_{\left[O, M_{1}\right]}\left(\xi_{2}\right), \varphi_{O, H_{1}^{\prime}}^{\prime}\left(\xi_{2}\right)$ on $\left[O, M_{1}\right],\left[O, M_{1}^{\prime}\right]$ respectively. Then $R \partial\left\{\varphi_{c_{2}}\left(\xi_{2}\right)-\right.$ $\left.\left[O, M_{1}\right] \cap\left[O, M_{2}\right]+\left[O^{\prime}, M_{1}^{\prime}\right] \cap\left[O^{\prime}, M_{2}^{\prime}\right]\right\}=0$, therefore $\boldsymbol{\varphi}_{c_{2}}\left(\xi_{3}\right)-\left[O, M_{1}\right] \cap\left[O, M_{2}\right]$ $+\left[O^{\prime}, M_{1}^{\prime}\right] \cap\left[O^{\prime}, M_{2}^{\prime}\right]=Z_{2}$ is a cycle.

$\dot{\varphi}_{c_{2}}\left(\xi_{2}\right)=\varphi_{\left[O, H_{1}\right]}\left(\xi_{2}\right)-\varphi_{\left[O, H_{1}^{\prime}\right]}^{1}\left(\xi_{2}\right)$ is analogous to (1), then we consider a third interior point $\xi_{3}$ of $\tau_{0}^{n}$ and similar relation as above and so on. Therefore we obtain the following relation:

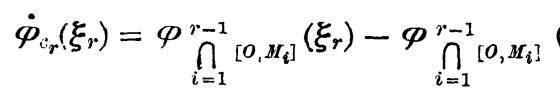

where $C_{r}$ is defined to be similar to $C_{2}$.

$$
\begin{aligned}
& \left.\left.\bigcap_{i=1}^{r}\left[O, M_{i}\right] \text { and } \bigcap_{i=1}^{r}\left[O^{\prime}, M_{i}^{\prime}\right] \text { are complexes bounded by } \varphi^{r-1} \bigcap_{i=1}^{r-1}\left[0, B_{i}\right]\right\} \xi_{r}\right) \text {, }
\end{aligned}
$$

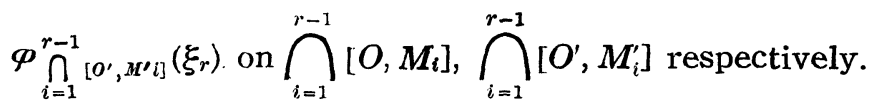

Then $R \partial\left\{\boldsymbol{\varphi}_{c_{r}}\left(\xi_{r}\right)-\bigcap_{i=1}^{r}\left[O, M_{i}\right]+\bigcap_{i=1}^{r}\left[O^{\prime}, M_{i}^{\prime}\right]\right\}=0$, and $\boldsymbol{\varphi}_{c_{r}}\left(\xi_{r}\right)-\bigcap_{i=1}^{r}\left[O, M_{i}\right]$ $+\bigcap_{i=1}^{r}\left[O^{\prime}, M_{i}^{\prime}\right]=Z_{r}^{n}$ is a cycle.

When we denote the projection of $Z_{r}^{n}$ on $S_{1}^{m}$ by $Z_{r}^{*}, Z_{r}^{n} \sim Z_{r}^{*}$ in $S^{n} \times I$. Then $F\left(Z_{r}^{n}\right) \sim f\left(Z_{r}^{*}\right)$ in $S^{n}$, therefore

$$
F\left(Z_{r}^{n}\right)=f\left({ }^{*}{ }_{r}^{n}\right) \text { in } S^{n} \text {. }
$$

On the other hand,

$$
\stackrel{*}{Z}_{r}^{n} \sim 0 \text { in } S_{1}^{n}
$$




$$
\begin{aligned}
& F\left(\stackrel{Z}{Z}_{r}^{*}\right)=f\left(\stackrel{Z}{Z}_{r}^{n}\right) \sim 0 \text { in } S^{n}, \\
& f\left(\ddot{Z}_{r}^{\prime \prime}\right)=0 \text { in } S^{n} .
\end{aligned}
$$

Therefore

$$
\begin{aligned}
& F\left(Z_{r}^{n}\right)=0, \\
& F\left(\phi_{i^{\prime}}\left(\xi_{r}\right)\right)-f\left(\bigcap_{i=1}^{r}\left[O, M_{i}\right]\right)+g\left(\bigcap_{i=1}^{r}\left[O^{\prime}, M_{i}^{\prime}\right]\right)=0, \\
& F\left(\phi_{c_{r}}\left(\xi_{r}\right)\right)=0, \\
& f\left(\bigcap_{i=1}^{r}\left[O, M_{i}\right]\right)=g\left(\bigcap_{i=1}^{r}\left[O^{\prime}, M_{i}^{\prime}\right]\right) \\
& W_{r}(f)=W_{r}(g) .
\end{aligned}
$$

3. In this chapter we shall investigate that $W_{r}(f)$ is used for determining whether a mapping of one sphere on another is essential or not. In case $m=n, W_{i}(f)$ means Brouwer degree and it is well known that $W_{r}(f)$ is only used for determining whether the mapping is essential or not. In case $m=$ $(r+1) n-r$ and $n$ is even, if $r=1, W_{r}(f)$ is the Hopf invariant and we showed in my preceding paper that the Hopf invariant is used for such purpose. In this chapter we consider the case $W,(f)$ is determined, that is (i) $m=n$, or (ii) $m=(r+1) n-r$ and $n$ is even. In the case (ii), by Lemma 2.4, $\bigcap_{i=1}^{r}\left[O, M_{i}^{m-\eta}\right]$ is a sum of finite manifolds which are at most of $n$ dimension having some singular simplexes at most of one-dimension. By a similar method as in Frendenthal's Lemma [2], we can consider that $\bigcap_{i=1}^{r}[O$, $\left.\boldsymbol{M}_{i}^{m-n}\right]$ is a manifold $\boldsymbol{M}^{n}$ which is at most of $n$-dimensions having some singular simplexes at most of one-dimention. Let $\tau_{0}^{n}$ be a fixed $n$-simplex and $\xi_{0}$ an interior point of $\tau_{0}^{n}, \xi_{0}$ be the antipodal point of $S^{n}$. We assume that $\sigma=\left(a_{0} a_{1} \ldots \ldots a_{n}\right), \sigma^{\prime}=\left(a_{0}^{\prime} a_{1} \ldots \ldots a_{n}\right)$ are oriented $n$-simplexes of $M^{n}$ with the common $(n-1)$-face $\tau=\left(a_{1} \ldots a_{n}\right)$. Then we obtain the following Lemma in my preceding paper.

LEMMA 3. 1. Let $f$ be a standard map of $M^{n}$ into $S^{n}$ and $f(\sigma)=+S^{n}, f\left(\sigma^{\prime}\right)$ $=\bar{\xi}_{0}$, then there is a standard map $g$ which is homotopic to $f$ and $g(\sigma)=\bar{\xi}_{0}$, $g\left(\sigma^{\prime}\right)=+S^{n}$ leaving the degree of $M^{i t}-\left(\sigma+\sigma^{\prime}\right)$ fixed.

LEMMA 3.2. Let $f$ be a standard map of $M^{n}$ into $S^{n}$ and $f(\sigma)=+S^{n} f\left(\sigma^{\prime}\right)$ $=-S^{n}$, then there is a standard map $g$ which is homotopic to $f$ and $g(\sigma)=$ $\bar{\xi}_{0}, g\left(\sigma^{\prime}\right)=\bar{\xi}_{0}$ leaving the degree of $M^{n}-\left(\sigma+\sigma^{\prime}\right)$ fixed.

A map $f$ of $S^{m}$ on $S^{n}$ may be considered a simplicial map of $K^{\prime \prime}$ on $\check{K}^{n}$. Let $P_{0}$ be an interior point of a fixed simplex $\tau_{0}^{n}$ of $\check{K}^{n}$. We may assume that inverse image $\phi_{S^{m}}\left(P_{v}\right)$ of $P_{0}$ for $f$ is a manifold $M^{m-n}$. The $m$ - 
dimensional simplexes $T_{a}^{m}$ of $K^{m}$ which are mapped on $\tau_{0}^{n}$. necessarily intersect $M^{m-n}$ and $M^{m-n} \cap T_{\alpha}^{m}$ are $(m-n)$-simplexes. $\xi_{i}(i=0,1,2, \ldots, r)$ in $\S 2$ may be considered in $\tau_{0}^{n}$ without any loss of generality. $M$-dimensional simplexes $T_{\alpha}^{m}$ of $K^{m}$ which are mapped on $\tau_{0}^{n}$ necessarily intersect $M_{1} \cap\left(\bigcap_{i=2}^{n}\left[O, M_{i}\right]\right)$ and $M_{1} \cap\left(\bigcap_{i=2}^{n}\left[O, M_{i}\right]\right) \cap T_{a}$ are $(r-1)$-simplexes. We denote it by $\left(\boldsymbol{a}_{0}^{\alpha_{k}}, \boldsymbol{a}_{1}^{\alpha_{k}}, \ldots, \boldsymbol{a}_{n-1}^{\boldsymbol{\alpha}_{k}}\right)$. Let $\left(\boldsymbol{e}_{0}^{\boldsymbol{\alpha}_{k}}, \boldsymbol{e}_{1}^{\alpha_{k}}, \ldots, \boldsymbol{e}_{n-1}^{\alpha_{k}}\right)$ be an $(n-1)$-face of $T_{\alpha}^{m-n}$ such that any two of its vertices are not mapped by $f$ on the same vertex of $\tau_{0}^{n}$, then $\left\{a_{0}^{\alpha_{k}}, a_{1}^{\alpha_{k}}, \ldots, a_{n-1}^{\alpha_{k}} \ldots, e_{0}^{\alpha_{k}}, \ldots, \boldsymbol{e}_{n-1}^{\alpha_{k}}\right\}$ is a $(2 n-1)$-simplex

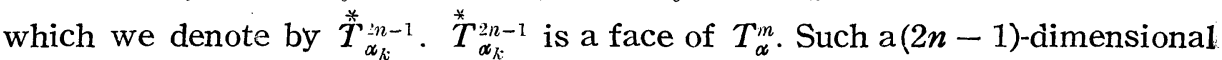
simplex $T_{\alpha_{k n}}^{*}{ }^{2 n-1}$ is considered for all $(n-1)$-dimensional-simplex $T_{\alpha_{k}}^{* 2 n-1}$ of $M_{1} \cap\left(\bigcap_{i=2}^{r}\left[O, M_{i}\right]\right) \cap T_{a}^{n}$. We consider all $(2 n-1)$-simplexes $T_{\alpha_{k}}^{* 2 n-1}$ which involve some $n$-simplex of $M_{1} \cap\left(\bigcap_{i=2}^{r}\left[O, M_{i}\right]\right)$. Then $\sum_{\alpha} \sum_{\alpha_{k}}\left(e_{0}^{\alpha_{k}}, \iota_{1}^{\alpha_{k}}, \ldots, e_{n-1}^{\alpha_{k}}\right)$ is $(n-1)$-manifold with singularity. We denote is by $M^{n-1}$ and consider a following complex :

$$
\begin{aligned}
& \left(\boldsymbol{a}_{0}^{\alpha_{k}} a_{1}^{\alpha_{k}} \ldots . a_{n-1}^{\alpha_{k}}, e_{0}^{\alpha_{k}}\left(+(-1)^{n}\left(\boldsymbol{a}_{1}^{\alpha_{k}}, \ldots, a_{n-1}^{\alpha_{k}}, \boldsymbol{e}_{0}^{\alpha_{k}}, \boldsymbol{e}_{1}^{\alpha_{k}}\right)+\ldots\right.\right. \\
& +(-1)^{n(n-1)}\left(a_{n-1}^{\alpha_{k}}, e_{0}^{\alpha_{k}}, \boldsymbol{e}_{1}^{\alpha_{k}}, \ldots, \boldsymbol{e}_{n-1}^{\alpha_{k}}\right) \text {. } \\
& \sum_{\alpha} \sum_{\alpha_{k}}\left[\left(\boldsymbol{a}_{0}^{\alpha_{k}} \boldsymbol{a}_{1}^{\boldsymbol{\alpha}_{k}} \ldots \ldots a_{n-1}^{\alpha_{k}}, e_{0}^{\alpha_{k}}\right)+(-1)^{n}\left(\boldsymbol{a}_{1}^{\alpha_{k}} \ldots, a_{n-1}^{\alpha_{k}}, \boldsymbol{e}_{0}^{\alpha_{k}}, \boldsymbol{e}_{1}^{\boldsymbol{\alpha}_{k}}\right)+\ldots\right. \\
& \left.\ldots+(-1)^{n(n-1)}\left(a_{n-1}^{\alpha_{k}}, e_{0}^{\alpha_{k}}, e_{1}^{\alpha_{k}}, \ldots, e_{n-1}^{\alpha_{k}}\right)\right]
\end{aligned}
$$

is $n$-dimensional manifold with some singular simplexes bounded by $M_{1} \cap$ $\left(\bigcap_{i=2}^{r}\left[O, M_{i}\right]\right)$ and $M^{n-1}$. We denote it by $\bar{K}_{0}^{n}$.

We replace $\left(e_{0}^{\alpha_{k}}, \boldsymbol{e}_{1}^{\alpha_{k}}, \ldots, \boldsymbol{e}_{n-1}^{\alpha_{k}}\right)$ by $\left(\boldsymbol{e}_{p}^{\alpha_{k}}, \boldsymbol{e}_{p+1}^{\alpha_{k}}, \ldots, \boldsymbol{e}_{n-1}^{\alpha_{k}}, \boldsymbol{e}_{0}^{\alpha_{k}}, \ldots, \boldsymbol{e}_{p-1}^{\alpha_{k}}\right)$, then

$$
\begin{gathered}
\sum_{\alpha} \sum_{\alpha_{k}}\left[\left(a_{0}^{\alpha_{k}}, \boldsymbol{a}_{1}^{\alpha_{k}}, \ldots, \boldsymbol{a}_{n-1}^{\alpha_{k}}, e_{p}^{\alpha_{k}}\right)+(-1)^{n}\left(a_{1}^{\alpha_{k}}, a_{2}^{\alpha_{k}}, \ldots, a_{n-1}^{\alpha_{k}}, e_{p}^{\alpha_{k}}, \boldsymbol{e}_{p+1}^{\alpha_{k}}\right)+\right. \\
\left.\ldots+(-1)^{n(n-1)}\left(a_{n-1}^{\alpha_{k}}, \boldsymbol{e}_{p}^{\alpha_{k}}, e_{p+1}^{\alpha_{k}}, \ldots, \boldsymbol{e}_{n-1}^{\alpha_{k}}, e_{0}^{\alpha_{k}}, e_{1}^{\alpha_{k}}, \ldots, e_{p-1}^{\alpha_{k}}\right)\right]
\end{gathered}
$$

is $n$-dimensional manifold with some singular simplexes bounded by $M_{1} \cap$ $\left(\bigcap_{i=?}^{r}\left[O, M_{i}\right]\right)$ and $M^{n-1}$. We denote it by $\bar{K}_{p}^{\prime}$. Since $M^{n-1}$ is homologous. zero in $S^{n}$, there exists an $n$-complex $\bar{K}^{n}$ bounded by $M^{n-1} . \quad K_{p}^{n}=\overline{K_{p}^{n}}+$ $\overline{\bar{K}}^{n}$ is an $n$-complex bounded by $M_{1} \cap\left(\bigcap_{i=2}^{r}\left[O, M_{i}\right]\right)$. As $p_{0}$. is an interior point of $\tau_{0}^{n}, p_{0}$ and vertices of each $(n-1)$-face of $\tau_{0}^{n}$ form $n$-simplexes $\tau_{0 c}^{n}$, $\tau_{1}^{n}, \ldots, \tau_{0 n}^{n}$. If we replace $\tau_{0}^{n}$ by $\tau_{c 0}^{n}, \ldots, \tau_{0 n}^{n}$ in $\ddot{\bar{K}}^{n}$, then the complex thus obtained is a finer simplicial subdivision than $\stackrel{*}{K}^{n}$. We may denote the 
resulted complex by the same notation $\ddot{K^{\prime}}$ for brevity, without any confusion. Similarly we can consider a fine simplicial triangulation of $K^{m}$ by inverse image of $\tilde{K}^{n}$ by $f$ and a suitable additional subdivision. We shall also denote it by $K^{m}$. $\left[O, M_{1}\right] \cap\left(\bigcap_{i=2}^{r}\left[O, M_{i}\right]\right)$ which is bounded by $M_{1} \cap\left(\bigcap_{i=2}^{r}\right.$ $\left[O, M_{i}\right]$ ) may be considered as one of $K_{p}^{n}$. When $M_{i}$ is mapped on $\xi$ by $f$ we denote the complex which is mapped on $\tau_{0}^{n}$ by $R\left(\xi_{i}\right)$. Evidently $R\left(\xi_{i}\right)=$ $\boldsymbol{R}\left(\xi_{j}\right) \quad(i \neq j, i . j=0,1,2, \ldots, r)$. We denote this common complex by $R$ and its $n$-skeleton by $R^{n}$.

THEOREM 3.1. If $W_{r}(f)=0$, then $f \mid R^{n}$ is homotopic to zero.

Proof. The map $f$ can be considered as a standard map without any loss of generality bv Lemma 2.1, when we use Lemma 3.1 and Lemma 3.2.

As $W_{r}(f)=0, \phi\left(M_{0}^{n}{ }^{n}, \bigcap_{i=1}^{n}\left[O, M_{i}\right]\right)=0$ and there is a set of $n$-simple$\operatorname{xes} \sigma_{1}, \sigma_{2}, \ldots, \sigma_{s} ; \sigma_{1}^{\prime}, \sigma_{i}^{\prime}, \ldots, \sigma_{s}^{\prime}$, on $\bigcap_{i=1}^{\prime}\left[O, M_{l}\right]$, where $\sigma_{i}$ and $\sigma^{\prime}$ are mapped on $S^{n}$ positively and negatively, respectively. $\bigcap_{i=1}^{r}\left[O, M_{i}\right]$ may be considered as one of $K_{p}^{n}$. For $\sigma_{i}$ and $\sigma_{i}^{\prime}(i=1,2, \ldots, s)$, there are regularly connected chain $\sigma_{i}+\sigma_{i_{1}}+\sigma_{i_{2}}+\ldots+\sigma_{i_{k}}+\sigma_{i}^{\prime}$ on $K_{p}^{n_{1}}$. It may be supposed that $d_{\mathcal{f}}\left(\sigma_{i_{1}}\right)=$ $+1, d_{f}\left(\sigma_{i_{1}}\right)=\ldots=d_{f}\left(\sigma_{i_{k}}\right)=0, d_{f}\left(\sigma_{i}^{\prime}\right)=-1$. Using Lemma 3.1, we deform $f$ in $\sigma_{i}+\sigma_{i_{1}}$, next in $\sigma_{i_{1}}+\sigma_{i_{2}}$, etc.; then using Lemma 3.2, we deform the map in $\sigma_{i_{k}}+\sigma_{i}^{\prime}$. The new map $f^{\prime}$ has as its degree $d_{f^{\prime}}\left(\sigma_{i}\right)=d_{f}\left(\sigma_{i_{1}}\right)=\ldots$

$=d_{f}\left(\sigma_{i}^{\prime}\right)=0$. We continue in this manner untill no simplexes are mapped positively and none are mapped negatively over $S^{n}$. Then $f \mid K_{p}^{n}$ is homotopic to zero, fixing the image of $M_{1}$ on $\xi_{1}$.

By Definition, $K_{p}^{n}=K_{n}^{n}+\bar{K}^{n}$ and $\bar{K}_{p}^{n}$ have no common $n$-simplex for any p. When $f$ is simplicial mapping, we consider the state where $K_{p}^{n}$ are mapped on $\tau_{00}^{n} . \overline{K_{p}^{n}}$ consist of the following complex :

$$
\begin{aligned}
& \left(a_{0}^{\boldsymbol{\alpha}_{k}}, a_{1}^{\alpha_{k}}, \ldots, a_{n-1}^{\boldsymbol{\alpha}_{k}}, \boldsymbol{e}_{p}^{\boldsymbol{\chi}_{k}}\right)+(-1)^{n}\left(\boldsymbol{\alpha}_{1}^{\alpha_{k}}, a_{2}^{\alpha_{k}}, \ldots, a_{n-1}^{\alpha_{k}}, e_{p}^{\alpha_{k}}, e_{p+1}^{\alpha_{k}}\right)+\ldots \\
& +(-1)^{n(n-1)}\left(\boldsymbol{a}_{n-1}^{\alpha_{k}}, \boldsymbol{e}_{p}^{\alpha_{k}}, \boldsymbol{e}_{p_{n+1}}^{\alpha_{k}}, \ldots, \boldsymbol{e}_{n n-1}^{\alpha_{k}}, \boldsymbol{e}_{0}^{\alpha_{k}}, \boldsymbol{e}_{1}^{\boldsymbol{\alpha}_{k}}, \ldots, \boldsymbol{e}_{p-1}^{\alpha_{k}}\right) \text {. }
\end{aligned}
$$

If $\left(\boldsymbol{a}_{n-1}^{\boldsymbol{\alpha}_{k}}, \boldsymbol{e}_{\boldsymbol{p}}^{\boldsymbol{\alpha}_{k}}, \boldsymbol{e}_{p_{+1}}^{\boldsymbol{\alpha}_{k}}, \ldots, \boldsymbol{e}_{n-1}^{\boldsymbol{\alpha}_{k}}, \boldsymbol{e}_{0}^{\boldsymbol{\alpha}_{k}}, \boldsymbol{e}_{1}^{\boldsymbol{\alpha}_{k}}, \ldots, \boldsymbol{e}_{p_{-1}}^{\boldsymbol{\alpha}_{k}}\right)$ is mapped on $\boldsymbol{\tau}_{00}^{n}$, other simplexes are mapped on faces of $\tau_{00}^{n}$ and their dimensions depend on numbers of $e_{i}$. $\left(a_{n-1}^{\alpha_{k}}, e_{n}^{\alpha_{k}}, e_{n+1}^{\alpha_{k}}, \ldots, \boldsymbol{e}_{n-1}^{\alpha_{k}}, \boldsymbol{e}_{0}^{\alpha_{k}}, e_{1}^{\alpha_{k}}, \ldots, \boldsymbol{e}_{p-1}^{\alpha_{k}}\right)$ is mapped on $\tau_{00}^{n}$ in the same manner for each $p$ except for orientation.

We may neglect this orientation when we take care of this similar property for all $\alpha_{k}$. If $\left(a_{n-1}^{\alpha_{k}}, \boldsymbol{e}_{p}^{\alpha_{k}}, \boldsymbol{e}_{p+1}^{\alpha_{k}}, \ldots, \boldsymbol{e}_{n-1}^{\alpha_{k}}, \boldsymbol{e}_{0}^{\alpha_{k}}, \boldsymbol{e}_{1}^{\alpha_{k}}, \ldots, \boldsymbol{e}_{n-1}^{\alpha_{k}}\right)$ is not mapped 
on $\tau_{00}^{n}$, we may neglect $T_{\alpha}^{m}$. In other words, we may consider that $\left(a_{0}^{\alpha_{k}}, a_{1}^{\alpha_{k}}\right.$, $\left.\ldots ., a_{n-1}^{\alpha_{k}}\right)$ contract tos a point and $T_{a}^{m}$ is empty. As $\overline{\overline{K^{n}}}$ is not mapped on $\tau_{00}^{n}$, we may consider only $\bar{K}_{p}$ for the degree based on $\tau_{00}^{n}$. On the other hand, deformations of Lemma 3.1 and Lemma 3.2 can bel introduced leaving the degree of $\bar{K}^{n}$ fixed. If we deform $f$ to the standard map $f^{\prime}$ by Lemma $2.1, f^{\prime}$ maps $\sum_{p} K_{p}^{n}$ on $\bar{\xi}_{0}$ and $f$ maps $R^{n}-\sum_{p} K_{p}^{n}$ on $\bar{\xi}_{0}$ by the above remark. Hence $f \mid R^{n} \simeq 0$.

When $M_{i}$ is mapped on $\xi_{i}$ by $f_{1}$ and $f_{2}$ we denote the complex which is mapped on $\tau_{0}^{n}$ by $R_{1}$ and $R_{2}$ respectively. We denote their $n$-dimentional skeleton by $R_{i}^{n}(i=1,2)$.

THEOREM 3.2. Let $f_{1}$ and $f_{2}$ be continuous mappings and $W_{r}\left(f_{1}\right)$ be equal to $W_{r}\left(f_{2}\right)$, then $f_{1}\left|R_{1}^{n}+R_{2}^{n} \simeq f_{2}\right| R_{1}^{n}+R_{2}^{n}$.

Proof. We consider Cartesian $(m+1)$-space $5^{m+1}$ and its subsets :

We and define $\varphi_{1}$ as follows :

$$
\begin{aligned}
& S^{m}=\left\{x \in\left(^{m+1} ; \sum_{i=1}^{m+1} x_{i}^{2}=1\right\},\right. \\
& E_{+}^{n}=\left\{x \in S^{n} ; x_{m+1} \geqq 0\right\}, \\
& E_{-}^{m}=\left\{x \in S^{m} ; x_{m+1} \leqq 0\right\}, \\
& S_{0}^{n-1}=\left\{x \in S^{m} ; x_{m+1}=0\right\} .
\end{aligned}
$$

$\varphi_{1}$ maps $E_{+}^{m} \quad$ onto $S^{m}$,

$\boldsymbol{\varphi}_{1}$ is a homeomorphism on $E_{+}^{m}-S_{0}^{m-1}$,

$\varphi\left(S_{0}^{m-1}\right)=P$, where $P$ is a fixed point on $S^{n}, d\left(\varphi_{2}\right)=1$.

We also define $\varphi_{2}$ as follows :

$\varphi_{2}$ maps $E_{-}^{m}$ onto $S^{m}$,

$\varphi_{2}$ is a homeomorphism on $E_{-}^{m}-S_{0}^{n-1}$,

$\boldsymbol{\varphi}_{2}\left(S_{0}^{m-1}\right)=P$,

$d\left(\boldsymbol{\varphi}_{2}\right)=-1$.

We may assume $f_{1}(P)=f_{2}(P)=Q$ without any loss of generality. We construct a map $F$ of $S^{m}$ into $S^{n}$ as follows:

$$
F= \begin{cases}f_{1} \varphi_{1} & \text { on } E_{+}^{m} \\ f_{2} \varphi_{2} & \text { on } E_{-}^{m}\end{cases}
$$

From $W_{r}\left(f_{1}\right)=W_{i}\left(f_{2}\right)$ we know $W_{r}(F)=0$. If we denote by $[F],\left[f_{1}\right],\left[f_{2}\right]$ the homotopy classes of $F, f_{1}$ and $f_{2}$ respectively, $[F]=\left[f_{1}\right]-\left[f_{2}\right]$.

By Theorem 3.1, $F \mid R_{1}^{n}+R_{2}^{n} \simeq 0$, then $f_{1}\left|R_{1}^{n}+R_{2}^{n} \simeq f_{2}\right| R_{1}^{n}+R_{2}^{n}$.

Pontrjagin theorem ${ }^{(i)}$ may be obtained from Theorem 3.2 as its special case when we put $r=1$, and $n=2$. For the proof, see my paper.

THEOREM 3.3. (Pontrjagin's theorem). If $f_{1}$ and $f_{2}$ are maps of $S^{3}$ on $S^{2}$ and $W_{1}\left(f_{1}\right)$ is equal to $W_{1}\left(f_{2}\right)$, then $f_{1}$ is homotopic to $f_{2}$. 
4. Freudenthal introduced the idea of suspension in the well known paper [2]. We shall investigate in this section some relations of the suspension and $W_{r}(f)$.

Let $f$ be a map of $S^{m}$ into $S^{n}$. We denote the equators of $S^{m+1}$ and $S^{n+1}$ by $S^{m}$ and $S^{n}$ respectively and extend $f$ to a map $E f$ of $S^{m+1}$ into $S^{n+1}$ as follows :

A point of $S^{m+1}$ is represented by $(P, \beta)$, where $P$ is a point of $S^{i n}$ and $-1 \leqq \beta \leqq 1$. Similarly, a point of $S^{n+1}$ is represented by $\left(P^{\prime}, \beta\right)$, where $P^{\prime}$ is a point of $S^{n}$ and $-1 \leqq \beta \leqq 1$. We define

$$
E f(P, \beta)=(f(P), \beta) \text {. }
$$

If $m+1=(r+1)(n+1)-r$, and $n+1$ is even, it is trivial that $W_{r}(E f)=0$. Secondly we investigate its inverse. We denote a subset of $\pi_{m+1}\left(S^{n+1}\right)$ whose elements have 0 as $W_{r}(f)$ invariant by $\left[\pi_{m+1}\left(S^{n+1}\right)\right]_{0}$.

THEOREM 4.1. $E\left(\pi_{n}\left(S^{n}\right)\right)=\left[\pi_{n+?}\left(S^{n+1}\right)\right]_{0}$.

Proof. We consider a map $f$ of $S^{m+1}$ into $S^{n+1}$ where $W_{r}(f)=0$. If we can prove that the inverse image of a point $P^{\prime}$ of $S^{n+1}$ consists of only one point $P$, we can prove this theorem as follows: Let $V_{1}^{m+1}$ be a closed neighborhood of $P$ on $S^{m+1}$ and $V_{2}^{m+1}$ be the closure of the complement of $V_{1}^{m+1}$. Then $f\left(V_{1}^{m+1}\right)$ or $f\left(V_{2}^{m+1}\right)$ does not completely cover $S^{n+1}$ and we can deform $f$ to a form of $E g$. In order to prove that the inverse image of $P^{\prime}$ consists of only one point it is sufficient to prove two following properties : $1^{\circ} \bigcap_{i=1}^{r}\left[O, M_{i}\right]$ is mapped on $P^{\prime}$ by $f_{1}$ which is homotopic to $f .2^{\circ} \bigcap_{i=1}^{r}\left[O, M_{i}\right]$ is contractible to one point on itself. We shall prove them. By $W_{r}(f)=0$, we have $\phi\left(M_{0}, \bigcap_{i=1}^{r}\left[O, M_{i}\right]\right)=0$. As we know in the proof of Theorem 3.1, the image of $\bigcap_{i=1}^{r}\left[O, M_{i}\right]$ can be contractible to a point fixing the image of $M_{i} \cap\left(\bigcap_{i=2}^{r}\left[O, M_{i}\right]\right)$. Then the image of $\bigcap_{i=1}^{r}\left[O, M_{i}\right]$ by $f_{1}^{\prime}$ can be considered to be $P^{\prime}$. At first we consider any point $R$ in distance $\rho \leqq \varepsilon$ of $\bigcap_{i=1}^{r}\left[O, M_{i}\right]$, where $\varepsilon$ is sufficiently small and denote by $Q$ the fixed point of the segment $P O$ for which $R Q / Q O=(\varepsilon-\rho) / \rho$. Let $\varphi(R, \tau)$ move linearly along the segment $R Q$ as $\tau$ move from 0 to 1 . For the point $R$ in distance $\rho \geqq \varepsilon$, we set $\varphi(R, \tau)=R$. We denote the inverse of $\varphi(R, \tau)$ by $\psi(R, \tau)$, and define $f_{1+\tau}=f_{1}\left(\psi(R, \tau)\right.$ ), then $f_{2}$ maps $O$ and only $O$ on $P^{\prime}$. The proof of (2) is complete.

We define some s pecial sets which are used for the proof of Theorem 4.2. 
Let $\left(5^{m+2}\right.$ be the Cartesian $(m+2)$ - space. We define its subsets as. follows :

$$
\begin{aligned}
& S^{m+1}=\left\{x \in\left(5^{m+2}: \sum_{i=1}^{m+2} x_{i}^{2}=1\right\},\right. \\
& S^{m}=\left\{x \in S^{m+1}: x_{m+1}=0\right\}, \\
& S_{\beta_{0}}^{m}=\left\{x \in S^{m+1}: x_{m+2}=\beta_{0}\right\}, \quad\left(-1 \leqq \beta_{0} \leqq 1\right) \\
& V^{m+2}=\left\{x \in\left(5^{m+2}: \sum_{i=1}^{m+2} x_{i}^{2} \leqq 1\right\},\right. \\
& E_{\beta_{0}}^{m+1}=\left\{x \in V^{m+2}: x_{m+2}=\beta_{0}\right\}, \\
& V_{\geq \beta_{0}}^{m+1}=\left\{x \in S^{n+1}: x_{m+2} \geqq \beta_{0}\right\}, \\
& V_{B_{0}}^{m+1}=\left\{x \in S^{m+1}: x_{m+2} \leqq \beta_{0}\right\} . \\
& V_{\beta_{1}, \beta_{2}}^{m+1}=\left\{x \in S^{m+1}: \beta_{1} \leqq x_{m+2} \leqq \beta_{2}\right\}, \\
&
\end{aligned}
$$

Similarly we define for $\left(^{n+2}\right.$.

THEOREM 4.2. If $m=(r+1) n-r$ and $n$ is even, $E$ is an isomorphism of $\left.\left[\pi_{m}\left(S^{n}\right)\right\}\right]$.

Proof. Let $g$ be a map of $S^{m}$ into $S^{n}$ and be $f=E g=0$. 'Then $f$ can be extended to a map of $V^{m+2}\left(S^{m+1}=R \partial V^{m+2}\right)$ into $S^{n+1}$. Let $\tau_{0}^{n+1}$ be a fixed simplex of $S^{n+1}$ and $\xi_{i}(i=1,2,3, \ldots, r)$ be the fixed interior points of $\tau_{0}^{n+1}$ and let $\xi_{i}$ exist on $S_{\beta}^{n}(-1<\beta<1)$. We denote the inverse image of $\xi_{i}$ in $S^{m+1}$ by $M_{i}^{m-n}$ and the inverse image of $\xi_{i}$ in $V^{m+2}$ by $Y_{i}^{m-n+1}$.

Then, we have

and

$$
f\left\{\bigcap_{i=1}^{r}\left[O, M_{i}\right]\right\}=W_{r}(f) S_{\beta_{0}}^{n},
$$

On the other hand $M_{i}^{m-n}$ belongs to $S_{\beta_{0}}^{m}$ essentially and there exists a complex $K_{i}^{m-n+1}$ bounded by $M_{i}^{m-n}$ in $S_{\beta_{0}}^{m}$. When we denote the fixed point of $S_{\beta_{0}}^{m}$ by $O$, we can consider $\left[O, M_{i}^{m-n}\right]$ as $K_{i}^{m-n+1}$. We define

$$
Z^{n}=Y_{1}^{m-n+1} \cap \bigcap_{i=2}^{r}\left[O, M_{i}\right]-\bigcap_{i=1}^{r}\left[O, M_{i}\right]
$$

There exist a complex $K^{n+1}$ which is bounded by $Z^{n}$ in $V^{m+2}$.

$$
\begin{aligned}
R \partial f\left(K^{n+1}\right)=f\left(R \partial K^{n+1}\right) & =f\left\{Y_{i}^{m-n+1} \cap\left(\bigcap_{i=2}^{r}\left[O, M_{i}\right]\right)\right\}-f\left\{\bigcap_{i=2}^{r}\left[O, M_{i}\right]\right\} \\
& =-W_{r}(f) S^{n},
\end{aligned}
$$

$f\left(K^{n+1}\right)$ covers $c^{\prime}$-times over $V_{\geqq ?_{0}}^{n+1}$ and $c^{\prime \prime}$-times over $V_{\geqq \beta_{0}}^{n+1}$.

$$
-W_{r}(f)=c^{\prime}-c^{\prime \prime} \text {. }
$$

$c^{\prime}$ and $c^{\prime \prime}$ have the following properties:

(i) $c^{\prime}$ and $c^{\prime \prime}$ are the same value when we use $Y_{i}^{n+1}(i=2,3, \ldots r)$. 
In the relation of $Z^{n}$ we replace $Y_{1}^{m-n+1}$ by $Y_{i}^{m-n+1}$, then it is similar to the proof of Lemma 2.8 that $c^{\prime}$ and $c^{\prime \prime}$ are invariant.

(ii) $c^{\prime}$ and $c^{\prime \prime}$ are not depend on a choice of $K^{n+1}$.

In $f\left(K^{n+1}\right)$ the difference of $K^{n+1}$ give us the difference anly of the image of bounding cycle.

(iii) $c^{\prime}$ and $c^{\prime \prime}$ do not depend on a choice of $O$. The proof is similar as in Lemma 2.7 .

(iv) $c^{\prime}$ and $c^{\prime \prime}$ do not depend on a choice of $\xi_{i}$. We shall prove it.

Let $\xi_{i}$ and $\stackrel{*}{\xi}_{i}$ be the fixed interior points of $\tau_{0}^{n+1}$, where $\xi_{i}$, $\xi_{i}^{*}$ exist on $S_{\beta_{0}}^{n}$ and $S_{\beta_{2}}^{n}$ respectively. We introduce the following complexes:

$$
\begin{aligned}
& M^{m-n}, \quad Y_{1}^{m-n+1},\left[O, M_{1}\right\rfloor, Z^{n}, K^{n+1} ; \\
& \ddot{\ddot{M}}^{m-n}, \quad \ddot{\hat{Y}}_{1}^{m-n+1},\left[\stackrel{*}{O}, \stackrel{*}{M_{1}}\right], \stackrel{*}{Z^{n}}, \stackrel{*}{K}^{n+1} \text {. }
\end{aligned}
$$

We consider a segment $x_{i}$ in $V_{\beta_{1}, \beta_{2}}^{n+1}$ whose end points are $\xi_{i}^{*}$ and $\xi_{i}$ and denote the inverse image of $x_{1}$ in $S^{m+1}$ by $Z_{12}^{m-n+1}$, the inverse image of $x_{1}$ in $V^{m+2}$ by $Y_{12}^{m-n+2}$. Besides we denote $V_{\beta_{1}, \beta_{2}}^{m+1}$ by $S^{n} \times I$ where $I$ is an interval $\beta_{1} \leqq t \leqq \beta_{2}$. We connect $O$ with $\stackrel{*}{O}$ by an arc in $S^{m} \times I$ which intersect with $S^{m} \times(t)$ on only one point. These points of intersection will be denoted by $O_{t}$. We also denote $x_{i} \cap S^{n} \times(t)$ by $\xi_{t t}$ in $S^{m} \times(t)$ by $M_{i t}$ and the inverse image of $\xi_{i t}$ in $E_{t}^{m+1}$ by $C_{i t}^{m-n+1}$. Then we have

$$
\begin{aligned}
& \operatorname{Ro}\left[Y_{12}^{m-n+2} \cap\left\{\bigcap_{\beta_{1} \leqq \leqq \beta_{2}}\left(\bigcap_{i=2}^{r}\left[O_{t}, M_{i t}\right]\right)\right\}\right] \\
& =\stackrel{Y}{Y}_{1}^{m-n+1} \cap\left(\bigcap_{i=2}^{r}\left[\stackrel{*}{O}, \stackrel{*}{M}_{i}\right]\right)-Y_{1}^{m-n+1} \cap\left(\bigcap_{i=2}^{r}\left[O, M_{i}\right]-\right. \\
& \left.Z_{12}^{m-n+1} \cap ! \bigcup_{\beta_{1} \leqq t \leqq \digamma_{2}}\left(\bigcap_{i=2}^{r}\left[O_{t}, M_{i t}\right]\right)\right\}
\end{aligned}
$$

and

$$
\begin{aligned}
& R \partial\left[Z_{12}^{m-n+1} \cap\left\{\bigcup_{\beta 1 \leqq 1 \leqq f_{2}}\left(\bigcap_{i=2}^{n}\left[O_{t}, M_{i t}\right]\right)\right\}\right] \\
& =\ddot{M}^{n-n} \cap\left(\bigcap_{i=2}^{r}\left[\stackrel{*}{O}, \stackrel{*}{M_{i}}\right]\right\}-M^{n-n} \cap\left(\bigcap_{i=2}^{r}\left[O, M_{i}\right]\right\}- \\
& \left\{\bigcup_{\beta 1 \leqq}\left(M_{i} \cap\left(\bigcap_{i=2}^{r}\left[O_{t}, M_{i t}\right]\right)\right\}\right. \text {. }
\end{aligned}
$$

We define

Then

$$
\boldsymbol{L}^{n+1}=\stackrel{*}{K^{\prime \prime}+1}-K^{n+1}-Y_{12}^{m-n+2} \cap\left\{\bigcup_{\beta_{1} \leqq \leqq \leqq S_{2}}\left(\bigcap_{i=2}^{r}\left[O_{t}, M_{i t}\right]\right)\right\} .
$$

$$
R \partial L^{n+1}=\stackrel{*}{Z}^{n}-Z^{n}+Y_{1} \cap\left(\bigcap_{i=2}^{r}\left[O, M_{i}\right]\right)-\stackrel{*}{Y}_{1} \cup\left(\bigcap_{i=2}^{r}\left[\stackrel{*}{O}, \stackrel{*}{M_{i}}\right]\right)+Z_{12}^{m-n+1}
$$




$$
\begin{gathered}
\left\{\bigcup_{\beta_{1} \leqq 1 \leqq \beta_{2}}\left(\bigcap_{i=2}^{r}\left[O_{i}, M_{i t}\right]\right)\right\} \\
=\bigcap_{i=1}^{r}\left[O, M_{i}\right]-\bigcap_{i=1}^{r}\left[\stackrel{*}{O}, \stackrel{*}{M_{i}}\right]+Z_{12}^{m-r+1} \cap\left\{\bigcup_{\beta_{1} \leqq i \leqq \beta_{2}}\left(\bigcap_{i=2}^{r}\left[O_{i}, M_{i t}\right]\right)\right\} .
\end{gathered}
$$

This complex is bounded by a cycle $M^{n+1}$ included in $V^{m+1}$. Therefore $R \partial$ $L^{n+1}=R \partial M^{n+1}$. By the fact of $M^{n+1} \subset V_{\beta_{1}, \ell_{2}}^{m+1}$, we know $f\left(M^{n+1}\right) \subset V_{\beta_{1}, \beta_{2}}^{n+1}$ and $f\left(M^{n+1}\right)$ cover the north pole and south pole with the degree 0 . As $n$ is even, $c^{\prime}+c^{\prime \prime}=0$, therefore $-W_{r} \cdot(f)=c^{\prime}-c^{\prime \prime}=2 c^{\prime}>0$.

To prove Theorem 4.2, using $W_{r}(f)=c^{\prime}=0$ and $E g=0$, it is sufficient to prove $g=0$. Now, by $W_{r}(f)=0$ we can deform $f$ in $S_{\beta}^{m}$ so as to $\operatorname{map} \bigcap_{i=1}^{r}\left[O, M_{i}\right]$ to one point and also $f\left(R \partial K^{n+1}\right)$ to one point. By $c^{\prime}=0$, the image of $K^{n+1}$ is contractible to one point fixing $R \partial K^{n+1}$ and also $K^{n+1}$ can be contractible to one point. Then, if $f\left(V^{m+2}\right) \subset S^{n+1}$, one point (for example north pole) has one point (for example north pole) and only one point as inverse image, therefore $g$ is not essential.

\section{Product theorem.}

THEOREM 5.1. Let $g$ be a map of one $m$-sphere $S_{1}^{m}$ into another $n$-sphere $S^{m}$ of degree c. If $f$ is a map of $S^{m}$ into $S^{n}$, then $W_{r}(f g)=c^{r} W_{r}(f)$.

Proof. As $c$ and $W \cdot(f)$ are constant for the homotopy class of maps respectively, we can assume that $f$ and $g$ are simplicial mappings. Let $\xi_{i}$ be a fixed point of $S^{n}$ and $\varphi\left(\xi_{i}\right)$ and $\psi\left(\xi_{i}\right)$ the inverse image of $\xi_{i}$ for $f$ and $f g$ respectively. We also assume that $m$-simplex $T^{m}$ of $S^{m}$ intersects with $\varphi\left(\xi_{i}\right)$ and the number of $m$-simplexes of $S_{1}^{m}$ mapped on $T^{m}$ positively or negatively by $g$ is $p$ or $q$ respectively. Then $p-q=c$ and the number of $(m-n)$-simplex of $\psi\left(\xi_{i}\right)$ are mapped on $(m-n)$-simplex of $\psi\left(\xi_{i}\right)$ which are included in $T^{m}$ positively or negatively is $p$ or $q$. Conversely the image of each $(m-n)$-simplex of $\psi\left(\xi_{i}\right)$ are $(m-n)$-simplex of $\phi\left(\xi_{i}\right)$. Therefore we have $g\left(\psi\left(\xi_{i}\right)\right)=c \cdot \varphi\left(\xi_{i}\right)$.

We define $L_{i}^{m-n+1}, K_{i}^{m-n+1}$ as follows :

$$
\begin{aligned}
& R \partial L_{i}^{m-n+1}=\psi\left(\xi_{i}\right), \\
& R \partial K_{i}^{m-n+1}=\phi\left(\xi_{i}\right) .
\end{aligned}
$$

Then,

$$
R \partial\left(g\left(\boldsymbol{L}_{i}^{m-n+1}\right)\right)=g\left(R \partial\left(\boldsymbol{L}_{i}^{m-n+1}\right)=g(\psi(\xi))=c \cdot \varphi(\xi)=c \cdot R \partial K^{m+n-1},\right.
$$

and, $g\left(L_{i}^{m-n+1}\right)-c \cdot K_{i}^{m-n+1}$ is a cycle of $S^{m}$. On the other hand, $g\left(L_{i}^{m-n+1}\right)-$ $c \cdot K_{i}^{m-n+1} \sim 0$ in $S^{m}$ and $f g\left(L_{i}^{m-n+1}\right)-c f\left(K_{i}^{m-n+1}\right) \sim 0$ in $S^{n}$.

Then, $f g\left(L_{i}^{m-n+1}\right)=c \cdot f\left(K_{i}^{m-n+1}\right)$.

As in the proof of Theorem 2.1, we may use $\left[O, \psi\left(\xi_{i}\right)\right],\left[O, \psi\left(\xi_{i}\right)\right]$ for $K_{i}^{m-n+1}, L_{i}^{m-n+1}$ respectively. Then $f g\left(\bigcap_{i=1}^{r}\left[O, \psi\left(\xi_{i}\right)\right]\right)=c^{r} \cdot f\left(\bigcap_{i=1}^{r}\right.$ 
$\left.\left[O, \varphi\left(\xi_{i}\right)\right]\right)$, therefore $W_{r}(f g)=c^{r} \cdot W_{r}(f)$.

THEOREM 5.2. Let $h$ be a map of one $n$-sphere $S^{n}$ into another $n$-sphere $S_{1}^{n}$ of degree $c$. If $f$ is a map of $S^{m}$ into $S^{n}$, then

$$
W_{r}(h f)=c^{r+1} W_{r}(f) .
$$

Proof. As $c$ and $W_{r}(f)$ are constant for the homotopy class of maps respectively, we can assume that $h$ and $f$ are simplicial mappings. Let $\xi$ be a fixed point of $S^{n}$ and $\phi$ and $\psi$ the inverse image for $f$ and $h f$ respectively. The inverse image of $\xi$ for $h$ are denoted by, $\eta_{1}, \ldots, n_{n} ; \zeta_{1}, \zeta_{2}$, $\ldots ., \zeta_{q} ;$ where $n$-simplexes including $\eta_{i}$ and $\zeta_{i}$ are mapped on $n$-simplex including $\xi$ positively and negatively respectively. It is clear that

$$
c=p-q \text { and } \quad \psi(\xi)=\Sigma \phi_{\left(\eta_{i}\right)}-\Sigma \varphi\left(\zeta_{j}\right) .
$$

We define $K_{i}^{m-n+1}, L_{j}^{m-n+1}$ as follows :

$$
\begin{aligned}
& R \partial K_{i}^{m-n+1}=\varphi\left(\eta_{i}\right) \\
& R \partial L_{j}^{m-n+1}=\varphi\left(\zeta_{j}\right) .
\end{aligned}
$$

Therefore we have

$$
R \partial\left(\sum K_{i}^{m-n+1}-\sum_{j} L_{i}^{m-n+1}\right)=\psi(\xi)
$$

$\sum K_{i}{ }^{-n+1}-\sum L_{j}^{m-n+1}$ is used for $W_{r}(h f)$, analogously

$\sum K_{i}^{m-n+1}-\sum L_{j}^{m-n+1}$ is used for $(p-q) W_{r}(f)$. The degree $c$ above considered is the degree of for $h$. Therefore we have.

$$
W_{r}(h f)=c^{r} \cdot c \cdot W_{r} \cdot(f)=c^{r+1} W_{r}(f) .
$$

\section{BIBLIOGRAPHY}

[1] K. AOKI, On maps of a $(2 n-1)$-dimensional sphere into an $n$-dimensional sphere. Tôhoku Math. Journ., 4(1952)

[2] H. FREUDENTHAL, UUber die Klassen der Sphären abbildungen, Compositio Math., 5(1937), 299-314.

[3] H. HOPF, Über die Abbildungen der dreidimensionalen Sphären auf die Kugelfläche, Math. Ann., 104(1931), 637-665.

[4] H. HoPF, Ưber die Abbildungen von Sphären auf Sphären niedrigere Dimension. Fund. Math., 25(1935), 427-440.

[5] H. HCPF, Die Klassen der Abbildiungen der $n$-dimensionalen Polyeder auf der $n$ dimensionale Sphären Comment. Math. Helv., 5(1933), 39-54.

[6] L. Pontruagin, A classification of mappings of the three dimensional complex into the two-dimensional sphere. Rec. Math., N.S., 9(1941), 331-363.

[7] H. WHITNEY, On the maps of an $n$-sphere into another $n$-sphere. Duke Math. Journ., 8(1937), 46-50.

[8] H. WHITNEY, The maps of an $n$-complex into au $n$-sphere. Duke Math. Journ., 3(1937), 51-55.

MATHEMATICAL INSTITUTE, TÔHOKU UNIVERSITY SENDAI. 